\title{
Nanoscale mapping and control of antenna- coupling strength for bright single photon sources
}

\author{
Anshuman Singh ${ }^{1}$, Pablo M. de Roque ${ }^{1}$, Gaëtan Calbris ${ }^{1}$, James T. Hugall ${ }^{1}$, and Niek F. van Hulst ${ }^{1,2}$ * \\ ${ }^{1}$ ICFO - The Institute of Photonic Sciences, The Barcelona Institute of Science and Technology, 08860 \\ Castelldefels (Barcelona), Spain \\ ${ }^{2}$ ICREA - Institució Catalana de Recerca i Estudis Avançats, 08010 Barcelona, Spain \\ *James.Hugall@ICFO.eu; Niek.vanHulst@ICFO.eu
}

Cavity QED is the art of enhancing light-matter interaction of photon emitters in cavities, with opportunities for sensing, quantum information and energy capture technologies. To boost emitter-cavity interaction, i.e. coupling strength $g$, ultrahigh quality cavities have been concocted yielding photon trapping times of $\mu$ s to ms. However, such high- $Q$ cavities give poor photon output, hindering applications. To preserve high photon output it is advantageous to strive for highly localised electric fields in radiatively lossy cavities. Nanophotonic antennas are ideal candidates combining low- $Q$ factors with deeply localised mode volumes, allowing large $g$, provided the emitter is positioned exactly right inside the nanoscale mode volume. Here, with nanometre resolution, we map and tune the coupling strength between a dipole nanoantennacavity and a single molecule, obtaining a coupling rate of $g_{\max } \sim 200 \mathrm{GHz}$. Together with accelerated single photon output, this provides ideal conditions for fast and pure non-classical single photon emission with brightness exceeding $10^{9}$ photons/sec. Clearly, nanoantennas acting as "bad" cavities offer an optimal regime for strong coupling $g$, to deliver bright on-demand and ultrafast single photon nanosources for quantum technologies.

Keywords: coupling strength; single photon emitter; nanoantenna; nanocavity; strong coupling.

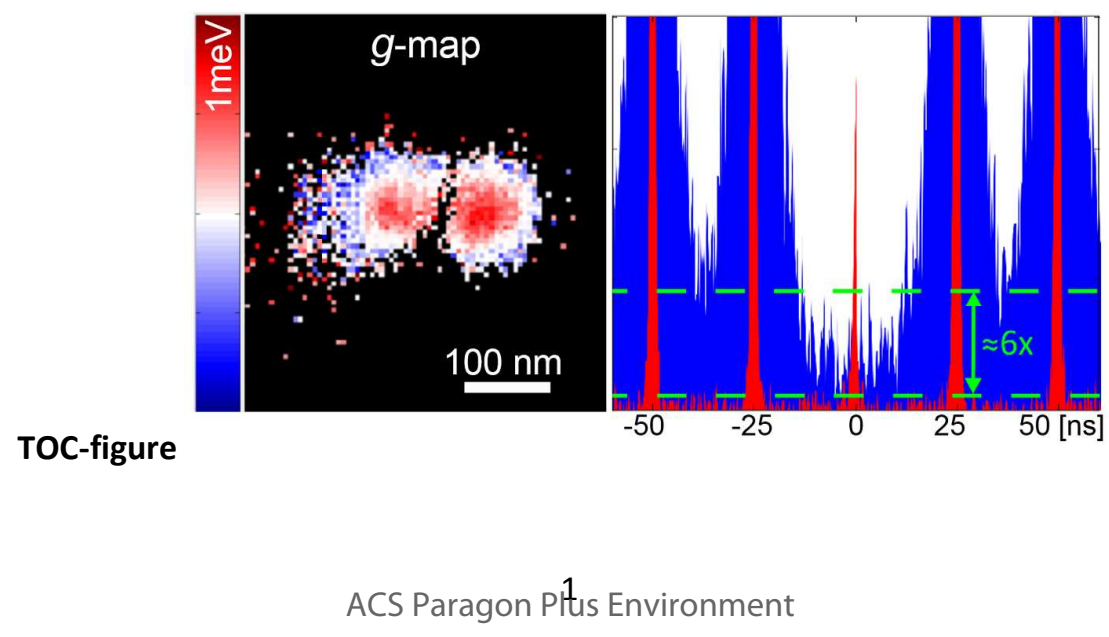


Photons play a key role as information carriers in quantum technologies, encoding and communicating quantum information in various degrees of freedom (polarization, wavelength, timing or path). Typically, scalable quantum computing schemes require efficient coherent interaction between weak single photon fields and matter. To boost this interaction, optical cavities can be used to tailor the optical field to strongly interact with the emitter ${ }^{1-7}$. Such cavity QED schemes achieve this coherence, known as strong coupling, using cavities with high quality factors $Q$ or small mode-volumes $\mathrm{V}$ (high photon density) where given a particular oscillator strength, the coupling strength scales as $g \propto 1 / \sqrt{V}$. Such high- $Q$ cavities generally require low temperatures and exhibit extremely sharp spectral windows. As such, and due to the large number of loss channels, coherence is notoriously difficult to maintain, preventing scalability to large quantum circuits at room temperature ${ }^{8}$. Alternatively, all-optical schemes based on linear-optical quantum computing ${ }^{9}$ or probabilistic entanglement ${ }^{10,11}$ are promising alternatives to such schemes, removing most of the burden of maintaining light-matter coherence, simplifying scalability ${ }^{12}$. Such all-optical systems rely principally on bright single photon sources with ideal characteristics: on-demand availability, limited frequency-bandwidth, emission into a defined spatio-temporal mode, and crucially high brightness corresponding to a large photon output rate ${ }^{13}$. Here we present a scheme for such a triggered, pure, ultrafast photon source at room temperature, based on a single molecule emitter deterministically coupled to a nanoantenna-cavity ${ }^{14-17}$ with high antenna efficiency, optimally tuned with nanometre precision for maximal coupling efficiency.

A single quantum emitter, such as a quantum dot, nitrogen vacancy centre (NV), molecule or single atom, spontaneously emits single photons, at a characteristic radiative rate $\Gamma_{0}$, as it transitions from an excited state to its ground state. Control of this emission rate can be achieved by coupling the system to an optical cavity, which itself has a decay rate $\kappa=\omega_{0} / Q$, which determines the cavity photon lifetime. One can reach distinctive regimes, depending on the strength of the coupling $g$, governed by the overlap and strength of the electric field $\boldsymbol{E}(\boldsymbol{r}, \omega)$ of the antenna and the effective dipole moment $\boldsymbol{p}$ of the emitter: $g=\boldsymbol{p} \cdot \boldsymbol{E}(\boldsymbol{r}, \omega) / \hbar$. When $g \gg>, \Gamma_{0}$, , the system is said to be 
strongly coupled, meaning that the excited state energy oscillates many times between the photon emitter and cavity before decaying. This has been achieved in high- $Q$ factor cavities, such as FabryPérot, whispering gallery and photonic crystal type cavities with low $\kappa .^{18}$. Although strong coupling in these cavities can be reached, the brightness of such systems is consequently low due to the low cavity decay rate $\kappa$ (Fig. 1).

The photon emission rate (PER) of a single photon source, at low excitation intensity is proportional to $\frac{\Gamma_{0}}{\Gamma_{0}+\Gamma_{\text {nonrad }}} \frac{\sigma}{h v} \mathrm{I}_{\mathrm{exc}}$, i.e. the quantum efficiency (ratio of radiative rate to sum of other rates), the absorption cross-section $\sigma$ and the excitation intensity $\left(\mathrm{l}_{\text {exc }}\right)$. For higher excitation intensity the PER will level off and the photon source will reach saturation with maximal PER proportional to the radiative rate $\Gamma_{0}$. In proximity of a nanoantenna cavity, the rates will be enhanced and as a result saturation will be reached at even higher excitation intensity and maximal PER. For a bright single photon source, the PER from the combined emitter-cavity system needs to be maximised, i.e. a short cavity photon lifetime. Therefore, the cavity decay rate $\kappa$, which puts a next limit on the maximum PER possible, should be kept as high as possible. To take advantage of this high $\kappa$, the coupling between the emitter and the cavity must also be tuned optimally $(g \sim \kappa)$ to ensure photons are efficiently emitted through this path. This can be achieved in the bad-cavity regime, where $\Gamma_{0} \ll g<\kappa .{ }^{20}$ In this regime, the combined decay rate of the system is approximately $4 g^{2} / \kappa$ at resonance (see Supporting Information Section A). This rate can be far superior to the emitter's free radiative rate $\Gamma_{0}$, with a large Purcell factor ${ }^{19}: F_{p}=1+4 g^{2} /\left(\kappa \Gamma_{0}\right)$. This large $F_{p}$ means that the photon is efficiently and rapidly emitted through the cavity mode. To increase $g$ sufficiently towards $\kappa$, we must increase the overlap of the emitter's dipole moment with the electric field mode of the cavity, by squeezing the cavity mode to smaller volumes. In dielectric cavities, however, the diffraction limit of light severely limits this and therefore other types of cavities should be sought (Fig 1.). 
Plasmonic nanoantennas make exemplary cavities for this type of coupling. In nanoantennas, light is coupled to electron oscillations that efficiently radiate to the far-field with high radiative rate and even good directivity ${ }^{9}$. Of equal importance, the cavity mode volume $V$ of nanoantennas, is extremely sub-wavelength, orders of magnitude below $(\lambda / n)^{3}$, providing large spatial overlap of the quantum emitter with the cavity mode, maximising $g$. Indeed strong Purcell enhancements of $10^{2}-10^{4}$ have been shown using plasmonic resonators ${ }^{21-24}$ and recently, the strong coupling regime has been reached with $F_{p} \sim 10^{6}$ at a $40 \mathrm{~nm}^{3}$ volume. ${ }^{25,26}$ Unfortunately, at such minute volumes, direct quenching to the metal of the emitter's excited state opens up an additional non-radiative loss channel, that severely limits the actual photon output (Fig. 1), despite the increase in spontaneous decay rate. Fortuitously, recently it was shown that mode hybridization in the strong coupling regime can yet again suppress the quenching and recover the emission ${ }^{27}$. Thus, given a nanoantenna cavity with high radiative efficiency and a nanoscale local mode volume, the main challenge becomes situating a single photon emitter at the accurate position within the mode volume for highest coupling strength $g$, while preserving the high photon emission rate and limiting any non-radiative losses ${ }^{28}$.

Here we present the deterministic positioning, with nm accuracy, of an oriented single molecule in the proximity of a dipole nanoantenna cavity, to create a nanoscale map of the coupling strength $g$. We reveal strong Purcell enhancement and a maximal $g$ value of $206 \mathrm{GHz}$, strongly dependent on the nanoscale position and orientation of the molecule within the cavity mode. By tuning the coupling, we demonstrate nanoantenna-controlled speeding-up of non-classical photon emission, enabling a bright and high-purity single photon source with ultrashort inter-photon times in the range of 100 ps.

Scanning a resonant nanoantenna over a single fluorescent molecule (Fig 2a), we obtain a fluorescence intensity map (Fig. 2 b) revealing the characteristic two-lobed pattern corresponding to the fundamental cavity mode of a dipole nanoantenna ${ }^{23}$. The mode is strongly localised at the ends of the nanoantenna with hotspot size of $20 \mathrm{~nm}$, producing a lateral mode confinement of $\approx 10^{3} \lambda^{2}$. 
We maintain an emitter-nanoantenna distance $>5 \mathrm{~nm}$ to prevent fluorescence quenching due to increase of the non-radiative rate $\left(\Gamma_{0}^{\text {nrad }}\right)^{24}$. The molecule is driven in the linear regime, far below saturation, emitting around $2 \times 10^{4}$ counts $/ \mathrm{sec}\left(\sim 10^{6}\right.$ photons $\left./ \mathrm{sec}\right)$, to allow sufficient time for a full molecule-cavity map before discrete photobleaching. At the hotspot centres, the fluorescence lifetime reduces from the free space value $\left(\tau_{0}=4.8 \mathrm{~ns}\right)$ to a minimum value of $\tau_{\text {tot }}=0.47 \mathrm{~ns}$ corresponding to a 10x enhancement in the spontaneous emission rate (Fig. $2 \mathrm{c}$ ). The increase in decay rate ( $\Gamma_{\text {tot }}$ ) is chiefly proportional to the increase in fluorescence intensity (Fig.2d) which confirms the predominantly radiative enhancement for our near-unity quantum yield fluorescent molecules. Still lifetime and intensity traces show some subtle asymmetries (Fig.2d); lifetime reduction is dictated by the overlap of emission dipole moment with local field vector at the emission wavelength, while intensity enhancement reflects the sum (interfering) of the local enhanced antenna near field and incident far field at excitation wavelength. For only slightly out-ofplane $x$-molecule, the lifetime/intensity response becomes asymmetric between both lobes.

On resonance and with $\Gamma_{0}<g<\kappa$, the contribution of the nanoantenna to the total decay rate is $\Gamma_{a n t}=4 g^{2} / \kappa$. The total emission rate of the molecule thus becomes $\Gamma_{t o t}=\Gamma_{a n t}+\Gamma_{0}^{r a d}+$ $\Gamma_{0}^{n r a d}=\Gamma_{a n t}+\Gamma_{0}$. We measure the ratio of the total decay rate $\left(\Gamma_{\text {tot }} \propto 1 / \tau_{\text {tot }}\right)$ with and without the nanoantenna $\left(\Gamma_{0}\right)$ in a single scan for one and the same molecule and relate it to the coupling strength $g$ through $\Gamma_{\text {tot }} / \Gamma_{0}=1+4 g^{2} /\left(\kappa \Gamma_{0}\right)$ (see Supporting Information Section A $)^{29}$. We extract the cavity loss rate $\kappa=8.8 \times 10^{13} \mathrm{~Hz}$ from the extinction spectrum of the nanoantenna with $Q$ factor $\approx$ 5 at a resonance wavelength of $680 \mathrm{~nm}$ (see Methods). Thus from the measured lifetime values we deduce $g$, and build a full $g$ map, basically with molecular resolution (Fig. 3a). The map shows values from $g=0$, in point 1 where the molecule is completely de-coupled from the cavity, and increases as the molecule enters the mode volume of the nanoantenna-cavity towards the hotspot (point 2) with a maximum value of $g_{\max }=206 \mathrm{GHz}(0.85 \mathrm{meV})$, and $g \gg \Gamma_{0}$. In this hotspot we are optimally positioned in the bad-cavity regime with a high coupling strength $g$ yet at same time with high $\kappa$, allowing efficient emission of photons from the cavity. The $g$ map shows good agreement 
with FDTD simulations (Fig. 3b), based on calculation of the power radiated from a dipole emitter in the near-field of a nanoantenna-cavity at a plane $15 \mathrm{~nm}$ below the nanoantenna surface.

Next we analyse the nano-positioning dependent photon statistics of the emitter, as it is controlled by $g$, especially at the hotspot where $g \gg \Gamma_{0}$. To this end we have determined the second-order intensity correlation function $g^{(2)}(\tau)$ throughout the $g$ map, as shown in Fig. 3c. At point 1 , free of coupling to the nanoantenna, the $\mathrm{g}^{(2)}(\tau)$ shows a normalized zero-time-delay coincidence rate well below the $\mathrm{g}^{(2)}(0)<0.5$ limit (Fig. 3c, blue curve 1 ), as expected for the non-classical single photon emission of the single molecule. The width of the correlation peaks is a direct measure for the molecular decay time. Measuring the photon statistics for increased coupling towards, i.e. moving the same molecule towards the hotspot (Fig. 3a, point 2), we see a dramatic sharpening of the $g^{(2)}(\tau)$ statistics, as the coupling increases, and the decay accelerates, to its fastest point at the hotspot centre (Fig. 3c, red curve). Interestingly, a marginal peak becomes visible at $g^{(2)}(0)$, as the very fast molecule-cavity decay causes rapid re-excitation of the molecule within one and the same excitation laser pulse (see Supporting Information Section C) ${ }^{30}$. The re-excitation and two-photon emission due to finite pulse length is known to affect single photon sources, while at the same time interesting for new multiphoton states ${ }^{31}$. The dramatic sharpening of $g^{(2)}(\tau)$ in the coupled case results from the 10-fold increase in the excited state decay rate and allows us to deduce that coupled single emitter-nanoantennas allow ultrashort inter-photon times in the range of 100 ps. When driven at saturation one can envisage bright $\left(>10^{9}\right.$ photons $\left.\cdot s^{-1}\right)$ true single photon sources.

A molecule, as a dipolar emitter within a vectorial electric field mode volume, is sensitive not only to position but also to the relative orientation. The orientation crucially affects the coupling strength between the cavity and the emitter as seen above where $g=\boldsymbol{p} . \boldsymbol{E}(\boldsymbol{r}, \omega) / \hbar$. The effect of this is already evident in the minor asymmetry observed in the coupling strength between the two nanoantenna hotspots visible in Fig. 3a, b. From this, we deduce slightly out-of-plane of the xoriented molecule. We note that mere intensity mapping (Fig. 2 b) is not sufficient, since intensity is susceptible to excitation conditions (including polarization and interference), whereas coupling 
strength is not. To illustrate the vectorial picture of the coupling strength $g$ we address cases of two single molecules of orthogonal orientation $(z, y)$ with respect to the nanoantenna long axis. Fig. $4 a$ and $4 \mathrm{~b}$ show calculated $g$ maps for $z$ - and y-molecule, resp. The fully out-of-plane z-molecule shows the dipolar two-hotspot pattern, similar to that seen for an in-plane molecule aligned along the antenna long axis (Fig. 3), however the coupling is even stronger due to the stronger z-fields between cavity and sample plane ${ }^{23,24}$. The in-plane $y$-molecule displays characteristic four-lobed pattern with weaker coupling (Fig. 4b). Experimentally the out-of-plane molecule is barely detectable without the nanoantenna, due to the difficulty of exciting out-of-plane molecules in a confocal setup. Yet, when coupled to the nanoantenna, both efficient excitation of the molecule, as well as emission into the antenna mode occur and indeed coupling $g$ up to $200 \mathrm{GHz}$ is detected (Fig. 4c). In-plane y-molecules, perpendicular to the antenna mode, are weakly enhanced and harder to find. Fig. 4d, e shows such case with the molecule largely along y yet a bit out-of-plane. The fourlobed pattern is a bit asymmetric character due to mixing in of coupling to the much stronger z-field. Clearly such nanometre precise, orientation resolved coupling maps allow the precise optimisation of a single emitter's placement to provide maximal radiative coupling and photon emission ${ }^{28}$.

To summarise we have mapped the coupling strength $g$ between a sub-wavelength sized nanoantenna and a single molecule with nanometre precision using differently orientated molecules to characterize the vectorial nature of the coupling strength $g$. The measured QED parameters for our nanoantenna-cavity are $\left(\Gamma_{0}, 2 g, \kappa\right)=(0.21 \mathrm{GHz}, 412 \mathrm{GHz}, 88 \mathrm{THz})$. We show that nanoantennacavities coupled to single photon emitters provide an optimal platform for super-bright and pure single photon sources due to their high radiative emission rate and sub-wavelength mode volumes allowing high molecule-cavity coupling strengths while providing fast photon emission rates.

Obviously, a bright emitter-nanocavity system requires a robust single photon source. Here we used photostable TDI molecules which allow driving the singlet-singlet photocycle beyond a $\mathrm{GHz}$, yet crossing to the triplet state, and ultimately photodissociation, limit such bright emission to durations of msec to second. Quantum dots are an alternative for bright photon emission ${ }^{32}$, but 
equally run into dark states and biexciton excitation at high power. Diamond NV centres are more photostable, yet less bright, while the diamond shell prevents close proximity to nanoantennas and limits the Purcell factor ${ }^{33}$. The novel single photon emitting defects in 2D-materials, such as boronnitride $^{34}$, are an interesting alternative, provided they live up to the required high power stability.

We underline the importance of the precise emitter position and orientation to maximise this coupling and thus photon emission rate. Such ultra-compact and directional single photon sources are highly compatible with integrated photonic devices and thus provide new opportunities in all-optical quantum information applications and solid-state devices. 


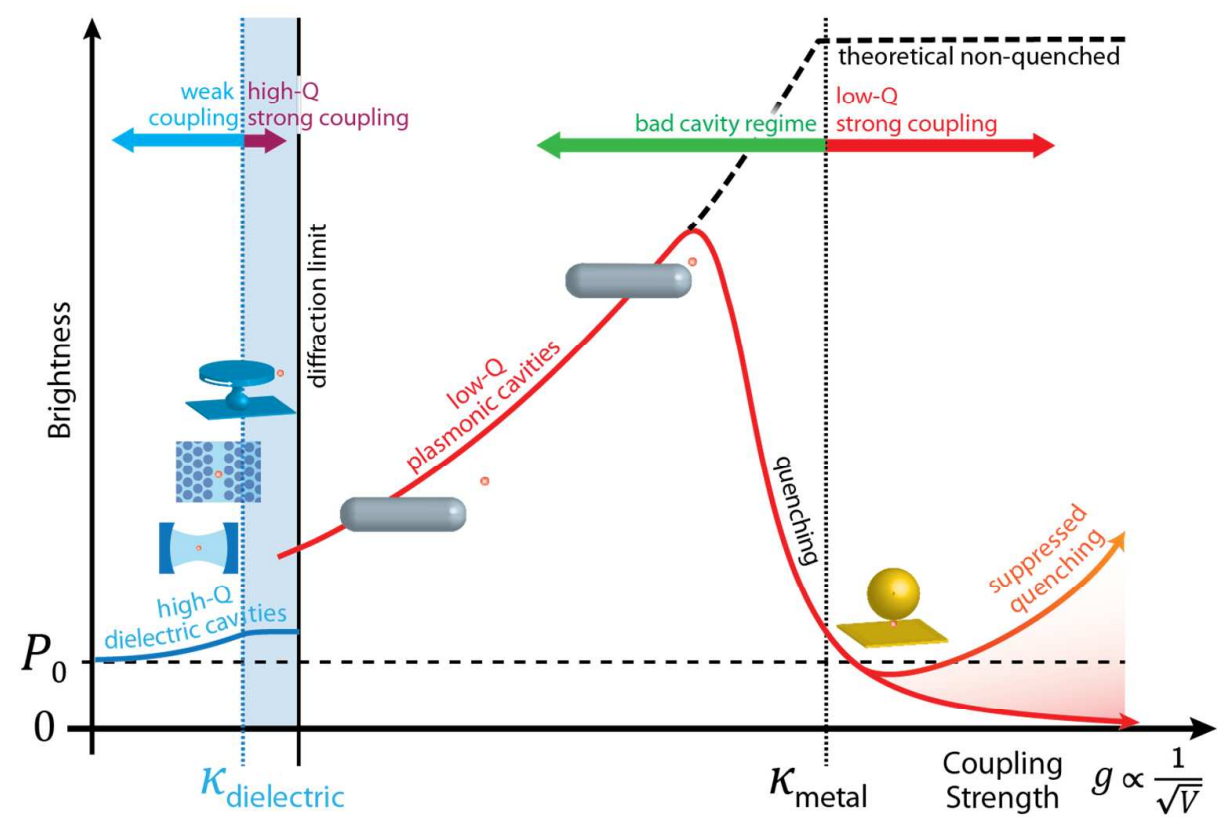

Figure 1. The optimal regime for strong coupling $g$ and bright photon emission for coupled

quantum emitter-cavity systems. Dielectric cavities, with diffraction limited mode-volumes [blue line], rely on high-Q factors to maximise coupling $g$ to a photon emitter. With such low losses ( $\left.\kappa_{\text {dielectric }}\right)$, however, their radiative efficiency and thus achievable brightness (photon emission rate) is limited. Plasmonic cavities [red line], are intrinsically low-Q, high loss cavities (high $\kappa_{\text {metal }}$ ), functioning as good photon radiators. Their sub-diffraction limited mode volumes allow for far greater coupling to the emitter thus permitting large increases in photon brightness. By careful design, their tiny mode-volumes can be pushed to maximise emitter coupling to the cavity and would reach a theoretical maximum photon brightness [black dashed line] as the strong coupling limit is reached, with coupling strength exceeding the cavity radiative loss rate $\kappa_{\text {metal }}$. However, at such small mode volumes $\left(<50 \mathrm{~nm}^{3}\right)$, proximity of the emitter to the metal surface, opens-up a nonradiative quenching channel to the metal, suppressing photon brightness below this theoretical limit. At even smaller volumes, in the strong coupling regime, new channels open up, suppressing the quenching. Maximal photon output is thus achieved in lossy plasmonic cavities in the bad-cavity regime, when coupling is maximised, but not beyond the point when quenching starts to dominate total photon output. 

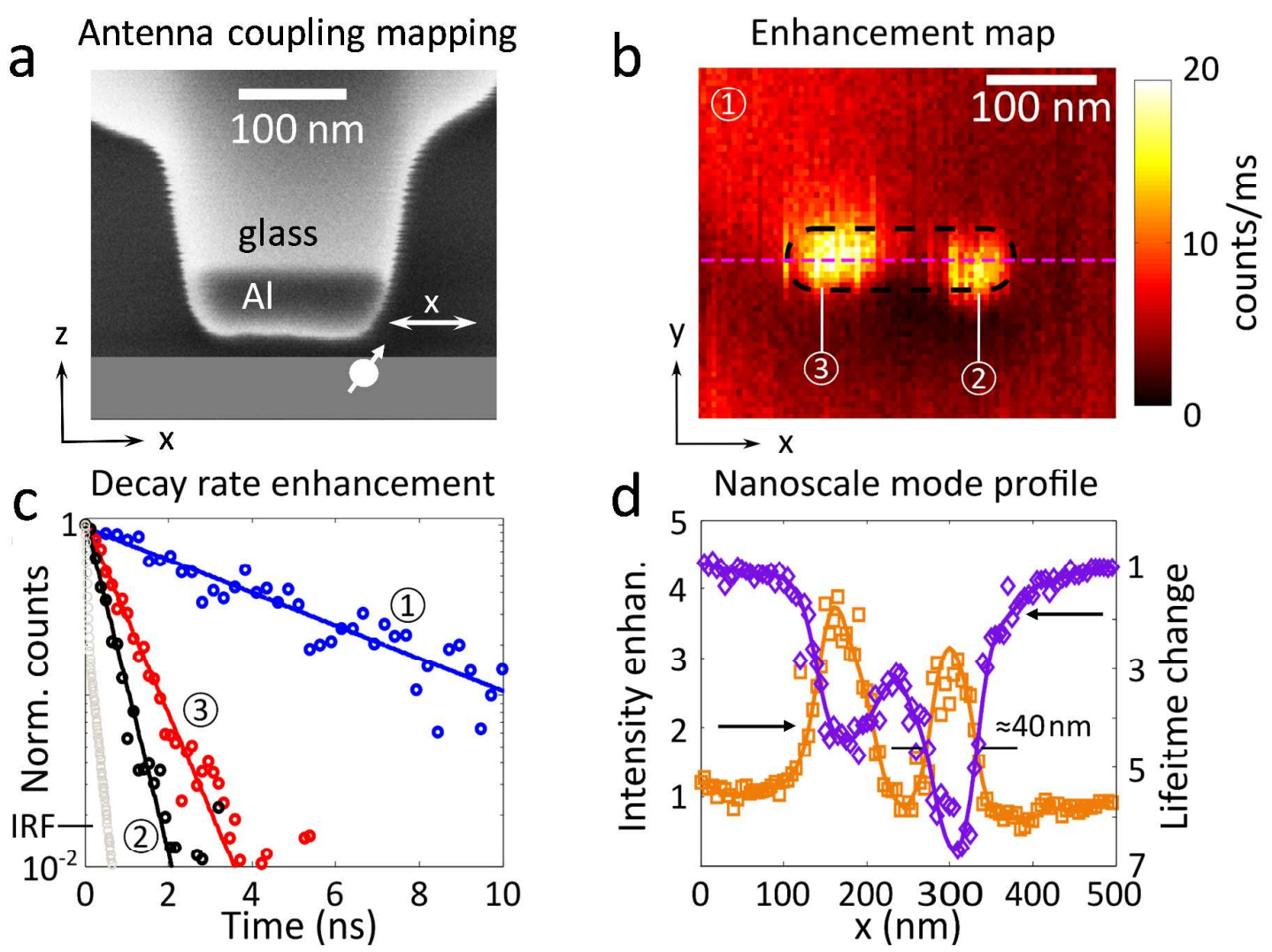

Figure 2. Emission and decay rate enhancement of the nanoantenna-cavity mode.

a, SEM image showing the aluminium dipole nanoantenna fabricated at the end of an optical fibre (see Methods section). The resonant nanoantenna-cavity is scanned $\sim 10 \mathrm{~nm}$ above a single fluorescent molecule, to capture the fluorescence lifetime and the $2^{\text {nd }}$ order correlation function of the single photon emitter at all positions of the nanocavity, with $5 \mathrm{~nm}$ step size, and retrieve a full map of the coupling strength $g$.

b, Intensity enhancement map as the nanoantenna-cavity is scanned over a single molecule revealing the two-hotspot dipolar mode of the cavity. The dashed black outline represents the size and geometry of the nanoantenna.

c, Characteristic fluorescence decay traces (recorded during lifetime imaging) of the same molecule both uncoupled (blue line) and coupled to the nanoantenna in the hotspots (red and black lines). The circles are data points and the solid lines are single exponential fits. The uncoupled molecule (1) has a lifetime of $\tau_{0}=4.79 \mathrm{~ns}$ which decreases to a minimum of $\tau_{\text {tot }}=470 \mathrm{ps}$ at hotspot (2), corresponding to a Purcell enhancement factor of 10 times. The grey curve indicates the instrument response function (IRF) of the time-tagging detection system. 
1

2

3

4

5

6

7

8

9

10

11

12

13

14

15

16

17

18

19

20

21

22

23

24

25

26

27

28

29

30

31

32

33

34

35

36

37

38

39

40

41

42

43

44

45

46

47

48

49

50

51

52

53

54

55

56

57

58

59

60

d, Line profiles of intensity and lifetime along the long axis of the antenna (dashed magenta line in b), show the correlated enhancement in fluorescence intensity (orange squares) and reduction in fluorescence lifetime (purple diamonds). The width of the nanoantenna cavity mode is as narrow as $40 \mathrm{~nm}$ FWHM, indicating strong lateral field localisation. The intensity and lifetime profiles show some mutual asymmetry, since the intensity is sensitive to the excitation conditions (near-field interference between incident field and vectorial antenna field, depending on molecular orientation), while the lifetime is only sensitive to emission of the molecule through the enhanced local density of states at the emission wavelength. 


\section{Figure 3. Optimizing coupling strength $g$-and photon statistics for a single molecule in a resonant} dipole nanoantenna-cavity.

a, Coupling strength $g$-map of the molecule-nanoantenna system with $5 \mathrm{~nm}$ step size. The map shows $g=0 \mathrm{GHz}$ away from the nanoantenna cavity, corresponding to zero coupling to the antenna, and reaches a maximum value in the antenna hotspots of $g_{\max }=206 \mathrm{GHz}(0.85 \mathrm{meV})$. The coupling strength varies rapidly within the nanoantenna hotspots due to the high field gradients, emphasising the need for precise nanoscale positioning of the single emitter in the nanocavity to optimize the coupling. The slight left-right asymmetry in the $g$-map is a direct consequence of the specific angle between the molecule's emission dipole and the nanoantenna field lines, with the overlap favoured on the right-hand side, corresponding to more efficient coupling to the nanoantenna.

b, Calculated coupling strength $g$-map of the molecule-nanoantenna system

c, Second-order photon cross-correlations $\mathrm{g}^{(2)}(\tau)$ (see Methods), where $\tau$ is the inter-photon arrival time delay, showing anti-bunched emitter character $\left[g^{(2)}(0)=0.097 \pm 0.010\right]$ of the single molecule when not coupled to the nanoantenna (point 1, blue). When coupled to the nanoantenna (point 2 , 
red) the molecule continues to show single photon character $\left[g^{(2)}(0)=0.13 \pm 0.02\right]$ yet with a strongly reduced lifetime manifested in the much narrower width of the correlation peaks.

d, An enlargement of the shaded area in the correlation curve in c shows at least a $6 x$ decreases in the photon-correlation noise level between two consecutive pulses, increasing the separability of emitted single photons at this excitation laser repetition rate. Moreover, the width of each correlation peak is $\sim 400$ ps FWHM, permitting a single photon source of rate $>1 \mathrm{GHz}$ while still maintaining sufficient purity. The new small $g^{(2)}(0)$ peak ( $\approx 10 \%$ of the side peaks) appearing in the coupled system is due to the rapid molecular decay compared to the pulse length of our excitation laser, causing multiple excitation and emission of the molecule, i.e. repumping within a single excitation pulse. (See Supporting Information Section C for a detailed discussion.) 


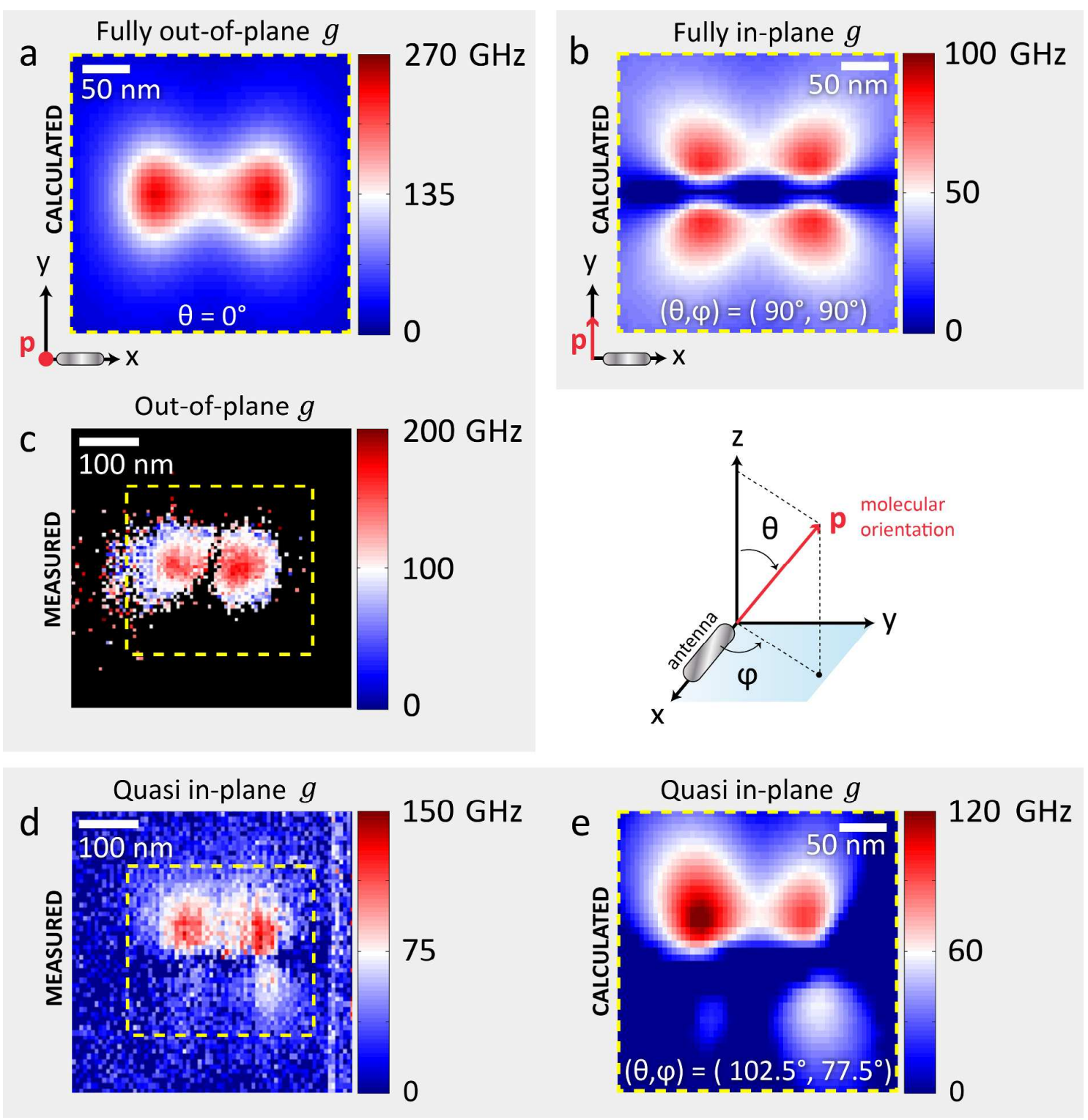

Figure 4. Vectorial mapping of the coupling strength $\boldsymbol{g}$. Measured and calculated coupling $g$-maps show how the coupling of the single molecule photon emitter to the nanoantenna-cavity depends both on the relative position (xyz) and orientation coordinates $(\theta, \phi)$ of the single molecule. Spherical coordinates $(\theta, \phi)$ with z perpendicular to the $x$-y sample plane and $x$ parallel to the antenna rod. The calculations show the $g$-maps for the area of interest, marked by the dashed square (yellow) in the experiment.

a, Calculated coupling map (from FDTD simulations of the radiated power, see Methods) for a molecule with out-of-plane z-orientation $\left(\theta=0^{\circ}\right)$, showing the familiar two-lobed dipolar pattern. The out-of-plane case shows higher coupling strengths than the in-plane case, with $g$ up to $\approx 241 \mathrm{GHz}$. 
b, Calculation for a molecule oriented in-plane along the $y$-axis $\left(\theta=90^{\circ}, \phi=90^{\circ}\right)$, perpendicular to the long antenna axis, demonstrating a characteristic (cross-polarization) four-lobed pattern and weaker coupling, up to $100 \mathrm{GHz}$, than x or z orientations;

c, Measured $g$-maps for out-of-plane $\mathbf{z}$ molecule. The emission is strongly enhanced when coupled to the antenna, yet further away without coupling the out-of-plane molecule gives insufficient photons for lifetime determination, and the $g$-value is set to black at these positions.

d, e, Measurement and calculation for a molecule with significant y-orientation component $\left(\theta=102.5^{\circ}, \phi=77.5^{\circ}\right)$ and mostly perpendicular to the long antenna axis, demonstrating a characteristic four-lobed pattern. A slight out-of-plane tilt of the y-oriented molecule towards the zaxis is responsible for the top-bottom asymmetry in the $g$-map, since the molecule emission couples more efficiently to the antenna mode when it is on one side of the nanoantenna. 


\section{Methods}

Single molecule sample preparation: Thin film single molecule samples are prepared by spin-coating a dilute $\left(10^{-9} \mathrm{M}\right)$ solution of terrylene diimide (TDI) dye molecules in $1 \%$ PMMA [poly(methyl methacrylate)] onto a cleaned glass coverslip. This provides a thin (<20 nm) layer of fluorescent molecules in the PMMA matrix, in which the molecules are spatially well separated (>2 $\mu \mathrm{m}$ molecule-molecule separation) and each of different discrete orientation with respect to the coverslip surface.

Nanoantenna-cavity fabrication: The nanoantenna-cavity is fabricated at the apex of a tapered optical fibre, which acts as support to attach the nanoantenna to a piezo-electric scanner and shear force feedback mechanism of the scanning antenna microscope. The nanoantenna is constructed out of a thin layer of evaporated aluminium (50 nm), sculpted into the correct geometry using Ga focused-ion-beam milling (FIB), forming a nanorod with a length of $175 \mathrm{~nm}$ and width and depth of $50 \mathrm{~nm}$. A detailed description of the fabrication process can be found in Ref. 23.

Nanoantenna-cavity resonance: The dimensions of the Al nanorod are chosen to act as a $\lambda / 2$ dipole nanoantenna-cavity, resonant at $680 \mathrm{~nm}$ with the emission of the TDI molecules and with $Q$ factor $\approx 5$. Figure 5 shows the dark field scattering spectrum of a single Al nanoantenna together with the emission spectrum of TDI. See also Supporting Information.

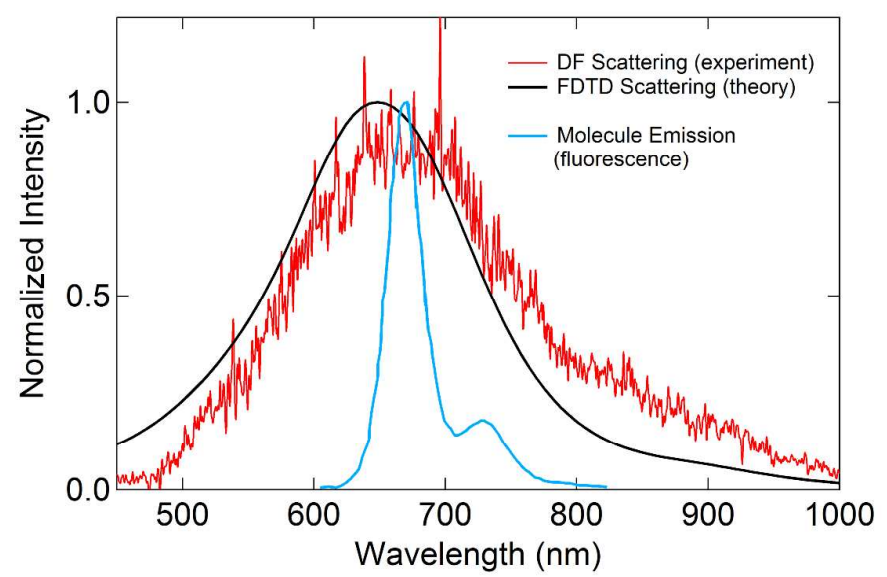

Figure 5: Normalized dark-field (DF) scattering spectrum of a single aluminium nanoantenna (in red) and theoretical FDTD modelling scattering spectra (black) compared to fluorescence emission spectrum of the molecule (in blue). 
Scanning antenna microscope: All experiments were performed using a custom-built scanning probe microscope head, on top of an inverted confocal microscope with piezo-electric scanners capable to scan both sample and antenna, and a shear-force feedback mechanism to control antenna-sample distance, as previously described ${ }^{23}$. Near-field intensity maps of the nanoantenna are acquired by scanning the nanoantenna with nanometre accuracy in the close vicinity $(\approx 10 \mathrm{~nm})$ of a fluorescent molecule, positioned in the diffraction-limited focus of the circularly polarized excitation laser beam $(\lambda=635 \mathrm{~nm})$ focused using a 100x 1.3NA oil-immersion microscope objective. The laser power before the objective never surpasses $2 \mu \mathrm{W}$, corresponding to energy densities below $4 \mathrm{~kW} / \mathrm{cm}^{2}$ at the focus. Under these imaging conditions, TDI molecules remain fluorescent for up to 30 minutes. In the collection path, a dichroic beam splitter and dielectric long pass filter are used to separate out the reflected excitation light, and the fluorescence is confocally imaged onto two fast avalanche photo-diodes (APD) to enable single photon counting and photon correlation.

Photon time-tagging (lifetime) maps: Single molecules are excited using a picosecond pulsed laser diode ( $\lambda=635 \mathrm{~nm}$, pulse width $<90$ ps, LDH-P-635, PicoQuant GmbH, Berlin) with 40 $\mathrm{MHz}$ repetition rate. Using a time-correlated single photon counting (TCSPC) correlator (PicoHarp 300, PicoQuant $\mathrm{GmbH}$, Berlin), we record the absolute arrival time of fluorescent photons on the APDs (PD-050-COD, Micro Photon Devices, Bolzano) relative to the excitation pulse from the laser with 8 ps resolution. The experimental instrument response function (IRF) is $\sim 40$ ps FWHM; see Fig. 2c). Using customised software and hardware, we synchronize the photon arrival time information with the scanner positioning the nanoantenna relative to the molecule. For each pixel (corresponding to a specific antenna-molecule position), the raw data is binned into $256 \mathrm{ps}$ arrival time windows from which we construct photon arrival histograms and extract the fluorescence lifetime. The constructed lifetime map allows us to build a coupling strength $g$-map of a single molecule coupled to the nanoantenna-cavity, for each position with $5 \mathrm{~nm}$ spatial resolution. 
Second-order photon cross-correlation measurements: We use a Hanbury-Brown-Twiss setup for second order photon correlation, where the collected signal is split 50/50 onto two APDs, both with instrument response times of 40 ps. The photon arrival times on the APDs are crosscorrelated in live histogramming mode, revealing the photon cross-correlation of the coupled system, for a given antenna-molecule position. The correlation histograms are recorded with a bin size of 64 ps and later binned at 256 ps as shown in Fig. 3c,d.

Numerical simulations: We solve Maxwell's equations using a finite-difference time domain (FDTD) commercial solver (Lumerical Solutions, Inc., Vancouver). The power radiated from a dipole emitter (molecule) placed in the near-field of a resonant Al nanoantenna $\left(170 \times 50 \times 50 \mathrm{~nm}^{3}\right)$ is calculated at a plane $15 \mathrm{~nm}$ below the nanoantenna surface (to match experimental conditions) and compared to the radiated power far away from the antenna (reference value). The ratio between the two radiated powers is equivalent to the ratio of emission rates. To construct the simulated coupling strength maps, the radiated power ratio is calculated at varying antenna-molecule positions in steps of $5 \mathrm{~nm}$ to match the experimental maps (Fig. 3). Moreover coupling $g$-maps are calculated for different orientations of the molecular emission dipole with respect to the sample surface and antenna (Fig. 4). The reference rate $\left(\Gamma_{0}\right)$ is different for in-plane and out-of-plane molecules due to the different effect of the glass-air interface on the molecule's spontaneous emission rate for each orientation $^{35}$. Based on our lifetime measurements of different molecules, we use an in-plane emission rate of $0.34 \mathrm{GHz}$ and $0.17 \mathrm{GHz}$ for the out-of-plane rate. 


\section{Acknowledgements:}

Authors would like to thank James Douglas and Darrick Chang for fruitful discussion on the physics of cavity QED; Sylwester Gawinkowski and Lisa Saemisch for spectroscopy and fabrication of aluminium nanoantennas. This work is supported by EU-project NanoVista (FP7-ICT-2011-7, No. 288263). N.F.v.H. acknowledges the financial support by the European Commission (ERC Advanced Grants 247330-NanoAntennas and 670949-Light-Net), Spanish MINECO (Projects FIS2015-69258-P, FIS201572409-EXP, Network FIS2014-55563-REDC/FIS2016-81740-REDC, and Severo Ochoa Grant SEV20150522), the Catalan AGAUR (2014 SGR01540), CERCA Programme of Generalitat de Catalunya and Fundació CELLEX (Barcelona).

\section{Author contributions:}

A.S., P.M.d.R. and J.T.H. designed and built the experiment. A.S. fabricated nanoantenna probes, performed the experiments and analysed the data. G.C. carried out simulations and numerical modelling. J.T.H., P.M.d.R. and N.F.v.H. wrote the manuscript. N.F.v.H. supervised the project.

The authors declare no competing financial interest

Supporting Information Available: [derivation of coupling strength, scattering and emission spectra, anti-bunching analysis]

\section{References:}

1. Goy, P.; Raimond, J. M.; Gross, M.; Haroche, S. Observation of cavity-enhanced single-atom spontaneous emission. Phys. Rev. Lett. 1983, 50, 1903-1906.

2. Thompson, R. J.; Rempe, G.; Kimble, H. J. Observation of normal-mode splitting for an atom in an optical cavity. Phys. Rev. Lett. 1992, 68, 1132-1135.

3. Yoshie, T.; Tang, L.; Su, S.-Y. Optical Microcavity: Sensing down to Single Molecules and Atoms. Sensors 2011, 11, 1972-1991.

4. Kimble, H. J. The quantum internet. Nature 2008, 453, 1023-1030.

5. Scully, M. O. Quantum photocell: Using quantum coherence to reduce radiative recombination and increase efficiency. Phys. Rev. Lett. 2010, 104, 207701.

6. Miller, R.; Northup, T. E.; Birnbaum, K. M.; Boca, A.; Boozer, A. D.; Kimble, H. J. Trapped atoms in cavity QED: coupling quantized light and matter. J. Phys. B At. Mol. Opt. Phys. 2005, 38, S551S565.

7. Khitrova, G.; Gibbs, H. M.; Kira, M.; Koch, S. W.; Scherer, A. Vacuum Rabi splitting in semiconductors. Nat. Phys. 2006, 2, 81-90. 
8. Yao, N. Y.; Jiang, L.; Gorshkov, A. V.; Maurer, P. C.; Giedke, G.; Cirac, J. I.; Lukin, M. D. Scalable Architecture for a Room Temperature Solid-State Quantum Information Processor. Nat. Comm. 2012, 3:800.

9. Knill, E.; Laflamme, R.; Milburn, G. J. A scheme for efficient quantum computation with linear optics. Nature 2001, 409, 46-52.

10. Cabrillo, C., Cirac, J. I., Garcia-Fernandez, P. \& Zoller, P. Creation of entangled states of distant atoms by interference. Phys. Rev. A 1999, 59, 1025-1033.

11. Delteil, A.; Sun, Z.; Gao, W.B.; Togan, E.; Faelt, S.; Imamoglu, A. Generation of heralded entanglement between distant hole spins. Nat. Phys. 2016, 12, 218-223.

12. Gao, W. B., Imamoglu, A., Bernien, H. \& Hanson, R. Coherent manipulation, measurement and entanglement of individual solid-state spins using optical fields. Nat. Photonics 2015, 9, 363373.

13. Kok, P.; Munro, W.J.; Nemoto, K.; Ralph, T.C.; Dowling, J.P.; Milburn, G.J. Linear optical quantum computing with photonic qubits. Rev. Mod. Phys. 2007, 79, 135-174.

14. Mühlschlegel, P.; Eisler, H.-J.; Martin, O. J. F.; Hecht, B.; Pohl, D. W. Resonant optical antennas. Science 2005, 308, 1607-1609.

15. Novotny, L.; van Hulst, N.F. Antennas for light. Nature Photonics 2011, 5, 83-90.

16. Singh, A.; Hugall, J. T.; Calbris, G.; van Hulst, N. F. Fiber-based Optical Nanoantennas for Single Molecule Imaging and Sensing. J. Light. Technol. 2015, 33, 2371-2377.

17. Schuller, J. A.; Barnard, E.S.; Cai, W.; Jun, Y.C.; White, J.S.; Brongersma, M.L. Plasmonics for extreme light concentration and manipulation. Nat. Mater. 2010, 9, 193-204.

18. Vahala, K. J. Optical microcavities. Nature 2003, 424, 839-846.

19. Purcell, E. M. Spontaneous emission probabilities at radio frequencies. Phys. Rev. 1946, 69, 681.

20. Ladd, T.D.; Jelezko, F.; Laflamme, R.; Nakamura, Y.; Monroe, C.; O'Brien, J.L. Quantum computers. Nature 2010, 464, 45-53.

21. Akselrod, G.M.; Argyropoulos, C.; Hoang, T.B.; Ciraci, C.; Fang, C.; Huang, J.; Smith, D.R.; Mikkelsen, M.H. Probing the mechanisms of large Purcell enhancement in plasmonic nanoantennas. Nat. Photonics 2014, 8, 835-840.

22. Kühn, S.; Håkanson, U.; Rogobete, L.; Sandoghdar, V. Enhancement of Single-Molecule Fluorescence Using a Gold Nanoparticle as an Optical Nanoantenna. Phys. Rev. Lett. 2006, 97, 017402.

23. Singh, A.; Calbris, G.; van Hulst, N.F. Vectorial nanoscale mapping of optical antenna fields by single molecule dipoles. Nano Lett. 2014, 14, 4715-4723.

24. Anger, P.; Bharadwaj, P.; Novotny, L. Enhancement and Quenching of Single-Molecule Fluorescence. Phys. Rev. Lett. 2006, 96, 113002.

25. Chikkaraddy, R.; de Nijs, B.; Benz, F.; Barrow, S.J.; Scherman, O.A.; Rosta, E.; Demetriadou, A.; Fox, P.; Hess, O.; Baumberg, J.J. Single-molecule strong coupling at room temperature in plasmonic nanocavities. Nature 2016, 535, 127-130.

26. Santhosh, K.; Bitton, O.; Chuntonov, L.; Haran, G. Vacuum Rabi Splitting in a Plasmonic Cavity at the Single Quantum Emitter Limit. Nat. Commun. 2016, 7:11823. 
27. Kongsuwan, N.; Demetriadou, A.; Chikkaraddy, R.; Benz, F.; Turek, V.A.; Keyser, U.F.; Baumberg, J.J.; Hess, O. Suppressed Quenching and Strong-Coupling of Purcell-Enhanced Single-Molecule Emission in Plasmonic Nanocavities. ACS Photonics 2018, 5, 186-191.

28. Hugall, J.T.; Singh, A.; van Hulst, N.F. Plasmonic Cavity Coupling, ACS Photonics 2018, 5, 43-53.

29. Meystre, P.; Sargent III, M. Elements of quantum optics. $4^{\text {th }}$ edn., Springer, 2007.

30. Brunel, Ch.; Lounis, B.; Tamarat, P.; Orrit, M. Triggered Source of Single Photons based on Controlled Single Molecule Fluorescence, Phys. Rev. Lett. 1999, 83, 2722-2725.

31. Fischer, K.A.; Hanschke, L.; Wierzbowski, J.; Simmet, T.; Dory, C.; Finley, J.J.; Vuckovic, J.; Muller, K. Signatures of two-photon pulses from a quantum two-level system, Nature Physics 2017, 13, 649-654.

32. Hoang, T.B.; Akselrod, G.M.; Argyropoulos, C.; Huang, J.; Smith, D.R.; Mikkelsen, M.H. Ultrafast Spontaneous Emission Source Using Plasmonic Nanoantennas. Nature Commun. 2015, 6:7788.

33. Bermúdez-Ureña, E.; Gonzalez-Ballestero, C.; Geiselmann, M.; Marty, R.; Radko, I.P.; Holmgaard, T.; Alaverdyan, Y.; Moreno, E.; García-Vidal, F.J.; Bozhevolnyi, S.I.; Quidant, R. Coupling of individual quantum emitters to channel plasmons, Nature Commun. 2015, 6:7883.

34. Tran, T.T.; Bray, K.; Ford, M.J.; Toth, M.; Aharonovich, I. Quantum emission from hexagonal boron nitride monolayers, Nature Nanotechnology 2016, 11, 37-41.

35. Kreiter, M.; Prummer, M.; Hecht, B.; Wild, U.P. Orientation dependence of fluorescence lifetimes near an interface. J. Chem. Phys. 2002, 117, 9430. 


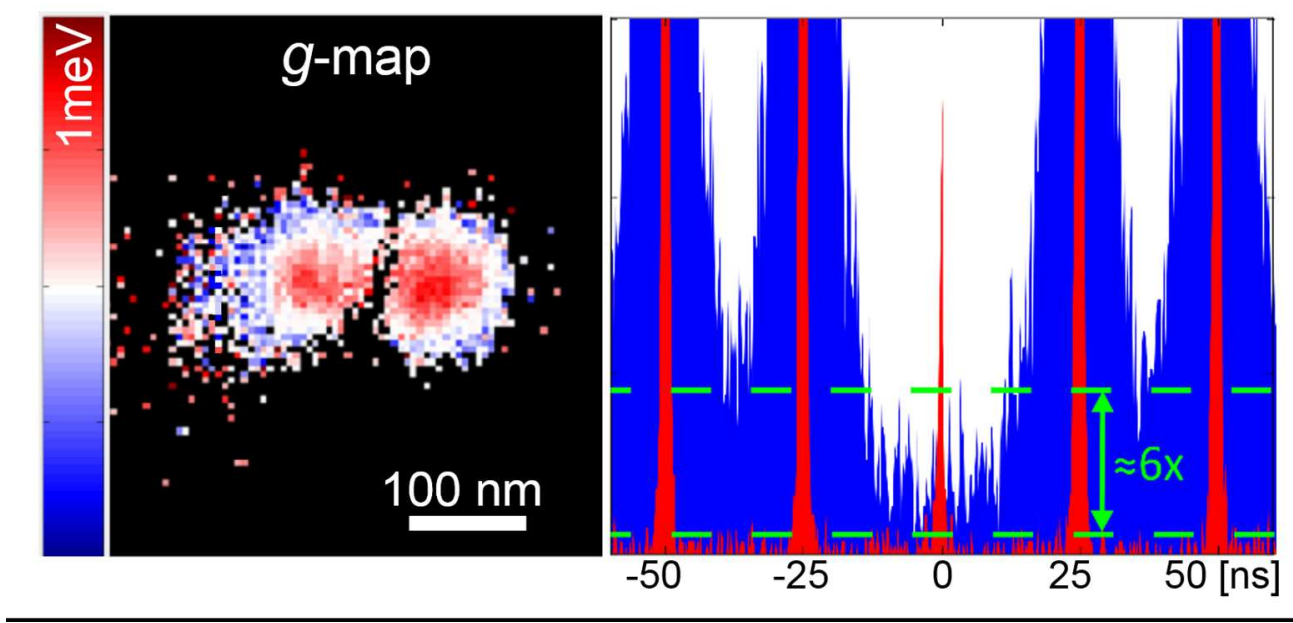

$282 \times 136 \mathrm{~mm}(150 \times 150 \mathrm{DPI})$ 